\title{
CAPACITACIÓN VIRTUAL Y SATISFACCIÓN PROFESIONAL MEDIANTE LA IMPLEMENTACIÓN DE TELECLÍNICAS DEL PROYECTO ECHO EN ECUADOR
}

\section{VIRTUAL TRAINING AND PROFESIONAL SATISFACTION BY IMPLEMENTING PROJECT ECHO TELECLINICS IN ECUADOR}

\author{
Lucila Jazmín De la Calle Andrade ${ }^{1}$ \\ María Angélica Barba Maggi² \\ Edwin Gilberto Choca Alcoser ${ }^{3}$ \\ Lizeth Estefanía Ortiz Terreros ${ }^{4}$ \\ Guillermo Gonzalo Gualpa Jaramillo
}

Ecuador

\section{RESUMEN}

En este artículo se ha evaluado el grado de satisfacción profesional del personal de salud rural, respecto a la capacitación por medio de teleclínicas del Proyecto ECHO. Se realizó un estudio descriptivo, transversal, de campo y no experimental. A través de la plataforma

1 Doctora en Medicina y Cirugía, Magister en Lingüística aplicada al aprendizaje del Inglés, Docente, Carrera de Medicina, Universidad Nacional de Chimborazo, Riobamba, Ecuador, Idelacalle@unach.edu.ec, https://orcid.org/0000-0003-23682027 Teléfono: (593) 33730880

2 Doctora en Química, Magister en Gestión Académica Universitaria, Docente, Carrera de Medicina, Universidad Nacional de Chimborazo, Riobamba, Ecuador, mbarba@unach.edu.ec, https://orcid.org/0000-0002-3056-2974 (593) 33730880

3 Doctor en Medicina y Cirugía, Especialista en Medicina Interna, Docente Carrera de Medicina, Universidad Nacional de Chimborazo, Riobamba, Ecuador, echoca@unach.edu.ec, https://orcid.org/0000-0003-4889-5395 (593) 33730880

$4 \quad$ Ingeniera en Biotecnología, Especialista de I+D+i, Corporación Ecuatoriana para el Desarrollo de la Investigación y la Academia CEDIA, Quito, Ecuador, lizeth.ortiz@cedia.org.ec, https://orcid.org/0000-0001-9113-6082 Teléfono: (593) 74079300 (593) 958864416

$5 \quad$ Doctor en Medicina y Cirugía, Especialista en Neurocirugía, Docente Carrera de Medicina, Universidad Nacional de Chimborazo, Riobamba, Ecuador, ggualpa@unach.edu.ec, https://orcid.org/0000-0003-1752-6642(593) 33730880 
Z00M, en las teleclínicas, se presentaron casos médicos de endocrinología e hipertensión, con la asesoría de especialistas y dirigido a profesionales de salud rural. Usando soporte digital gratuito, se aplicó un cuestionario de encuesta a 57 participantes, estructurado en seis dimensiones. Se efectuó la medición en base a la Escala de Likert; el análisis estadístico consistió en la obtención de la media y desviación estándar, así como porcentajes generales de satisfacción. La mayoría de participantes $(81,5 \%)$ estuvieron satisfechos con la aplicación de las teleclínicas. En todas las dimensiones se observaron puntuaciones que reflejan un elevado nivel de satisfacción. Desde la percepción de los profesionales, las teleclínicas les brindaron un soporte de aprendizaje permanente a bajo costo, contribuyendo al desarrollo profesional y la solución de problemas de la práctica médica.

PALABRAS CLAVE: Satisfacción, capacitación, teleclínicas, salud rural

\section{ABSTRACT}

This article presents the evaluation of professional satisfaction of rural health personnel, regarding the training through the teleclinics of Project ECHO. A descriptive, crosssectional, non-experimental and field study was performed. Through the ZOOM platform, medical cases of endocrinology and hypertension were presented in teleclinic sessions, with the advice of specialists and aimed at rural health professionals. Using free digital support, a survey questionnaire was applied to 57 participants, structured in six dimensions. The measurement was made based on the Likert Scale; the statistical analysis consisted of obtaining the mean and standard deviation, as well as general satisfaction percentages. Most participants $(81.5 \%)$ were satisfied with the application of the teleclinics. Scores that reflect a high level of satisfaction were observed in all dimensions. From the perception of the rural professionals, the teleclinics provided a permanent learning support at low cost, contributing to professional development and problem solving in the medical practice.

KEY WORDS: Satisfaction, training, teleclinics, rural health

\section{INTRODUCCIÓN}

Los sistemas de salud actuales incorporan varios principios que garantizan los derechos de la población, tales como: universalidad, equidad, integridad en la atención, entre otros. Existen múltiples reformas que buscan una mejora en la calidad de vida y satisfacción de beneficiarios, así como de prestadores de servicios de salud, a través de la descentralización y participación activa.

La Organización Mundial de la Salud en su declarativa universal, establece que uno de sus objetivos en común para los países es mejorar la salud de las personas en todo el mundo, con aliados estratégicos, organismos gubernamentales y no gubernamentales (Organización Mundial de la Salud, 2006).

La Constitución del Ecuador vigente desde el 2008, en el Art. 32, establece que la salud es un derecho garantizado por el Estado, “... mediante políticas económicas, sociales, culturales, educativas y ambientales; y el acceso permanente, oportuno y sin exclusión a programas, acciones y servicios de promoción y atención integral de salud, salud sexual y salud reproductiva..." (Asamblea Consitituyente, 2008, pág. 16).

"Los cambios sociales ocurridos en los últimos años en Ecuador han propiciado transformaciones radicales en el sistema de salud, destacándose el Modelo de Atención Integral de Salud Familiar, Comunitario e Intercultural (MAIS-FCI), que centra su accionar no solo en el individuo sino también en la familia y en la comunidad, con un enfoque predominante hacia la promoción de salud y la prevención 
de enfermedades, logrando la equidad en la prestación de los servicios y el respeto a la interculturalidad" (Naranjo Ferregut, Delgado Cruz, Rodríguez Cruz, \& Sánchez Pérez, 2014, pág. 380).

Según el Anuario Estadístico de América Latina y el Caribe, con información disponible a diciembre del 2018 mediante metodologías y estándares internacionales, en el Ecuador la población total proyectada es de 16.863.000, con un porcentaje de 64,4 que corresponde a población urbana y 35,6 de población ubicada en zonas rurales (Comisión Económica para América Latina y el Caribe CEPAL, 2018).

De acuerdo al Instituto Nacional de Estadísticas y Censos (INEC), en Ecuador existieron 21 médicos por cada 10.000 habitantes en el año 2016 (Instituto Nacional de Estadísticas y Censos INEC, 2016). Al analizar esta tendencia estadística, sumado a las limitadas condiciones de infraestructura y recursos tecnológicos para cubrir los requerimientos de servicios de salud, surge como estrategia primordial, la educación continua en aspectos administrativos, pero sobre todo en las competencias médicas específicas, lo que resulta de difícil acceso en zonas alejadas.

En Ecuador, desde 1970, se ha incluido el año de salud rural, con médicos, odontólogos, enfermeros y obstetrices, graduados de las universidades del país, quienes son asignados en las plazas establecidas por el Ministerio de Salud Pública (MSP), a través del levantamiento de necesidades por parte de las direcciones distritales de Salud. Estos profesionales se vinculan con los equipos de salud en Costa, Sierra, Oriente y Región Insular, cumpliendo también, en un alto porcentaje, con los cursos en línea establecidos por el MSP.

Entre los diversos problemas de la salud rural, es notoria la dificultad para el traslado de pacientes para interconsultas, las distancias entre los centros de salud o de atención primaria a unidades de mayor complejidad es amplia, no existe agenda de especialistas por la gran demanda, el paciente no cuenta con recursos económicos, en ciertos casos no tiene el apoyo familiar, sus condiciones físicas o psicológicas no son las mínimas requeridas para el traslado, ente otros aspectos.

Vía teleconferencia, es posible presentar los casos clínicos y ser sometidos a un análisis por especialistas, contribuyendo al diagnóstico, tratamiento in situ y seguimiento oportuno, todo enmarcado dentro del campo de acción del médico general o familiar.

El desarrollo tecnológico favorece el mejoramiento de la calidad de vida de la población. En el campo médico, existe un notable avance y desarrollo de diferentes equipos con tecnología de punta, para diagnóstico, tratamiento, investigación y comunicación.

En Ecuador no se ha podido evidenciar, normativa que legisle la aplicabilidad de la telemedicina, lo que se convierte en un requerimiento. "La telemedicina es una de las formas de superar las barreras de atención médica para los sitios alejados, aun así, es necesario invertir en infraestructura física y tecnológica para poder aplicarla" (Maruri Sigüenza \& Castro Rugel , 2016, pág. 39).

En el aspecto social, la telemedicina generaría beneficios para la comunidad y mejoraría la capacidad de atención médica, ya que se volvería accesible para personas que no puedan trasladarse o movilizarse a la ciudad por presentar cuadros graves (Lascano Soriano \& Sánchez Castro, 2018).

En el campo de la educación médica, se han desarrollado varias metodologías que hacen uso de herramientas digitales, aprovechando las imágenes, videos, sonidos, textos, que se han convertido en recursos virtuales 
convenientes para el mejoramiento del aprendizaje, principalmente en áreas de estudio complejas.

La gran revolución académica permite proveer de educación continua a profesionales médicos y de otras áreas de forma presencial o virtual. Los diseños curriculares de grado y posgrado, desarrollan competencias en el uso de la Tecnología de la información y comunicación (Tic), de la misma forma en el desempeño profesional, con una aplicabilidad amplia; adicionalmente, un alto porcentaje de las poblaciones cuentan con conexiones vía internet fijo o móvil, para facilitar comunicaciones locales, nacionales $y$ mundiales.

Ante esta realidad, el modelo de teleclínicas, basado en el Proyecto ECHO lacrónimo de Extension for Community Healthcare Outcomes), se presenta como una solución accesible para los médicos recién graduados que se encuentran ejerciendo su año de medicatura rural, así como para otros profesionales de los equipos de salud en áreas alejadas.

El Proyecto ECHO, desarrollado por la Facultad de Medicina de la Universidad de Nuevo México, es un modelo de capacitación a largo plazo, con actividades dirigidas, que transforma la educación médica y desarrolla vertiginosamente la competitividad en el trabajo para ofrecer un ejercicio profesional que extienda el manejo a nivel de especialidades, disminuyendo así las diferencias o discriminación en los servicios de salud. El núcleo del modelo ECHO TM es su sistema de socialización de conocimientos, encabezado por grupos de especialistas que utilizan videoconferencias en diferentes puntos, para realizar sesiones virtuales de clínicas a personal comunitario de salud, para mejorar la calidad de la atención a los pacientes en sus propias comunidades, reduciendo los costos. Durante las clínicas de teleECHO, se utiliza la tecnología disponible para teleconferencias
(University of New Mexico. School of Medicine., 2018).

En total, diversos grupos de 37 países están involucrados en el Proyecto ECHO, existen 14 centros (superhubs) y 650 programas ECHO (University of New Mexico, 2019).

En el año 2011, un estudio publicado por Sanjeev Arora y su equipo en The New England Journal of Medicine, marcó el inicio del desarrollo del Proyecto ECHO; se demostró que este modelo, es una opción efectiva para el tratamiento de infección por virus de la hepatitis $C$ en comunidades menos atendidas (Arora, y otros, 2011).

Actualmente, el modelo ECHO incluye la telecapacitación sobre más de 100 enfermedades o condiciones clínicas. Actúan más de 100 universidades, entre ellas Harvard y el MD Anderson en Estados Unidos, así como la Universidad de la República en Uruguay, pionero en Latinoamérica en aplicar esta metodología. En Estados Unidos, se ha logrado el apoyo de varias organizaciones privadas y gubernamentales. El Congreso de Estados Unidos aprobó en el 2017. de manera unánime un proyecto de ley para valorar y poner en actividad esta metodología (Cohen, 2019).

En Ecuador, con fundamento legal en el Convenio para Desarrollo de Proyecto ECHO entre la Corporación Ecuatoriana para el Desarrollo de la Investigación y la Academia CEDIA lentidad que mantiene el acuerdo de uso de licencia para las actividades del Proyecto ECHOl y la Universidad Nacional de Chimborazo (UNACH) (Corporación Ecuatoriana para el Desarrollo de la Investigación y la Academica CEDIA. Universidad Nacional de Chimborazo UNACH., 2018), con el propósito de solucionar las dificultades para la capacitación del personal de salud en áreas rurales, se ha implementado de manera continua el programa de teleclínicas a través de la plataforma Z00M, en el área de endocrinología e hipertensión, con 
la participación de la Carrera de Medicina de la UNACH y otras universidades.

Las reuniones virtuales cumplen aspectos administrativos, organizacionales, evolutivos, técnicos, metodológicos y funcionales (Ramón, y otros, 2014), sobre todo se enmarcan en el Proyecto ECHO. Las sesiones de una hora de teleclínica, tienen fundamento en el aprendizaje cooperativo, con la participación de médicos especialistas como asesores y capacitadores, y profesionales del área de salud, que se encuentran ejerciendo sus actividades en zonas rurales; la estructura de las teleclínicas implica la declaración de objetivos, la presentación de un caso clínico por parte de uno de los médicos que labora en áreas rurales, guardando rigurosamente las normas bioéticas; seguida de la exposición de preguntas y la resolución de las mismas, a través de la asesoría de los médicos especialistas. Posteriormente, se abre un espacio de intercambio de información entre todos los participantes, que involucra la estrategia de comunicación en tiempo real. Finalmente, se programa una fecha para evaluación y seguimiento (Figura 1).

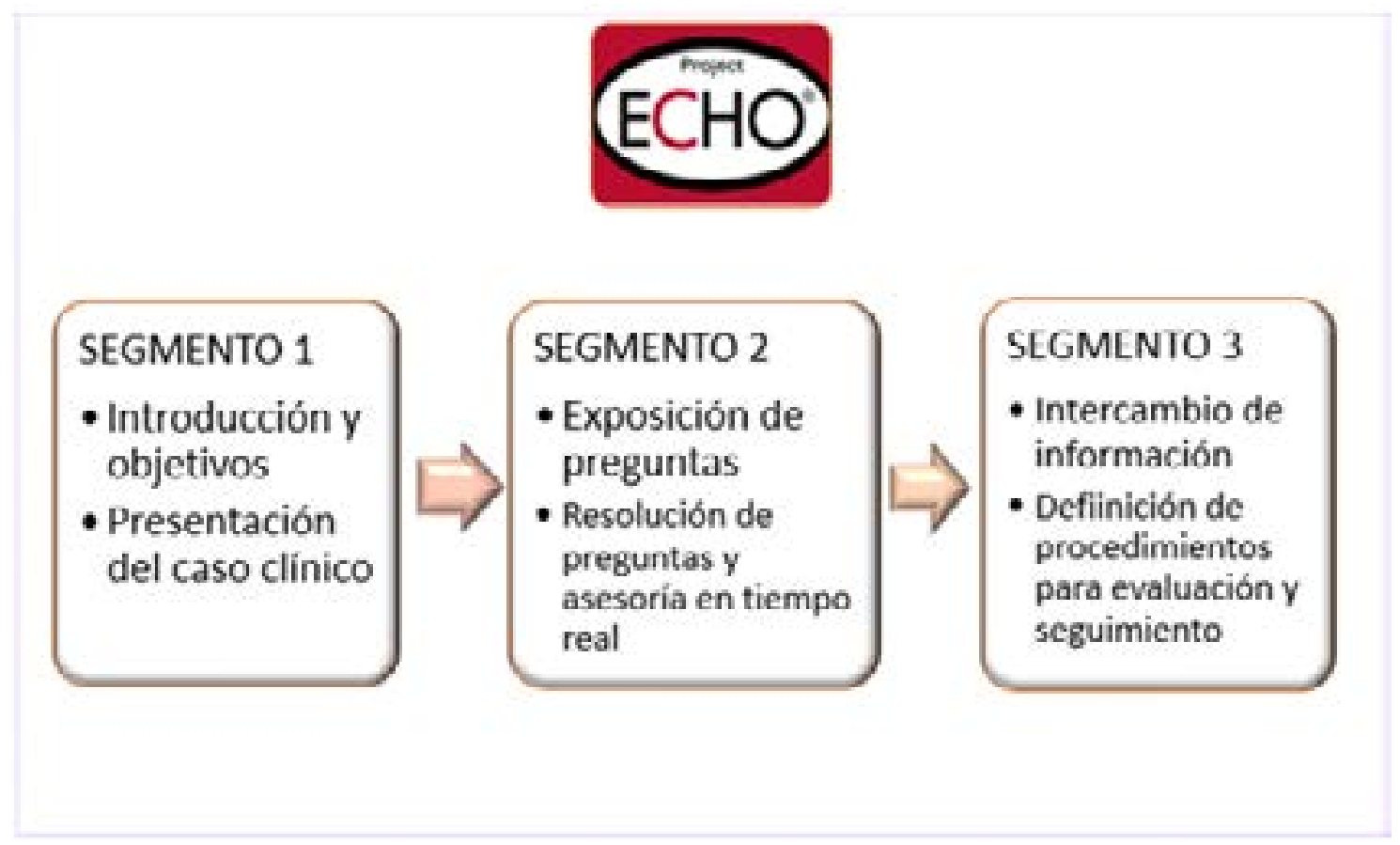

Figura 1. Modelo de acción de una sesión regular de teleclínica. Proyecto ECHO. Ecuador, 2018.

Fuente: Elaboración propia

De acuerdo a la información proporcionada en la página web, la plataforma Zoom de videoconferencias y mensajería, ayuda a las organizaciones a trabajar en equipo, a través de dispositivos móviles, computadoras de escritorio y otros (Zoom Video Commuications, Inc., 2019).

Las capacitaciones virtuales ofrecen ventajas, entre ellas: desaparición de distancias físicas, interactividad, comunicación controlada de forma sincrónica y asincrónica, trabajo cooperativo, aprendizaje personalizado, resolución inmediata de problemas, desconcentración del conocimiento y registro continuo de actividades (Marín Díaz , Reche Urbano, \& Maldonado Berea, 2013).

Las metodologías de capacitación on line, e-learning $o$ incluso b-learning, presentan algunos aspectos negativos y desafíos, tales como: desigualdad en el acceso a recursos tecnológicos, lo que conlleva la posibilidad de incurrir en la brecha digital, desacertada presunción sobre un adecuado manejo de 
la información y tecnología por parte de los participantes, entre otros (Cabero-Almenara, Llorente-Cejudo, \& Puentes-Puente, 2010).

Diversos estudios relacionados con el uso de Tic, muestran una gran aceptación estudiantil. Tal es el caso de la investigación realizada en la Escuela de Medicina "Luis Razetti" de la Universidad Central de Venezuela (UCV), respecto a la satisfacción estudiantil con el uso de estrategias basadas en las TIC; se encontró que todas las estrategias utilizadas fueron percibidas como satisfactorias, registrando la mayor aceptación el Campus Virtual con un 97,1\% (Díaz, Miguel, Landaeta, Ortiz, \& Fernández, 2014).

Las TIC tienen un importante rol para la creación de situaciones y entornos de aprendizaje, que fortalezcan el entrenamiento, el desempeño y el desarrollo de las competencias profesionales (Herrero Martínez, 2014).

Este tipo de educación ha sido útil también en la formación de profesionales, como lo demuestran Cordero y Caballero (2015), en su estudio sobre la aplicación de un formato semipresencial mediante aulas virtuales, en un curso de soporte vital avanzado dirigido a profesionales, dentro del programa de Enseñanza de Soporte Vital en Atención Primaria de la Sociedad Española de Medicina de Familia y Comunitaria (semFYC) (Cordero Torres \& Caballero Oliver, 2015).

Por todo lo expuesto, se ha desarrollado el presente estudio, que tiene como objetivo evaluar el grado de satisfacción profesional del personal de salud rural, sobre la aplicación del proceso de enseñanza a distancia y capacitación del Proyecto ECHO (teleclínicas) y su contribución en la solución de problemas en el manejo de los pacientes.

La evaluación del grado de satisfacción y la incidencia de estas capacitaciones en el tratamiento, diagnóstico y seguimiento de pacientes desde la percepción de los participantes, apuntan al mantenimiento de la calidad de los servicios profesionales desarrollados a través del Proyecto ECHO, fortaleciendo, además, la integración de redes académicas y de investigación, que contribuyen en el mejoramiento de los problemas del medio.

\section{MÉTODO}

Para el desarrollo de las teleclínicas, previo el diseño del cronograma respectivo, se han efectuado las convocatorias mediante correo electrónico para asegurar la participación de los profesionales de salud, así como tres especialistas en las ramas de Endocrinología y Medicina Interna.

El "Formulario de presentación de casos de Teleclínicas", es un documento delineado de acuerdo a los estándares del proyecto, proporcionado por CEDIA. En el mismo constan los siguientes acápites: Identificación del caso, información del presentador, información básica del paciente, historial médico del paciente, medicamentos, anamnesis sistémica, anamnesis familiar, examen físico, actividad física, recordatorio de la dieta, diagnósticos y laboratorio, preguntas principales para la teleclínica acerca del paciente (Figura 2). 


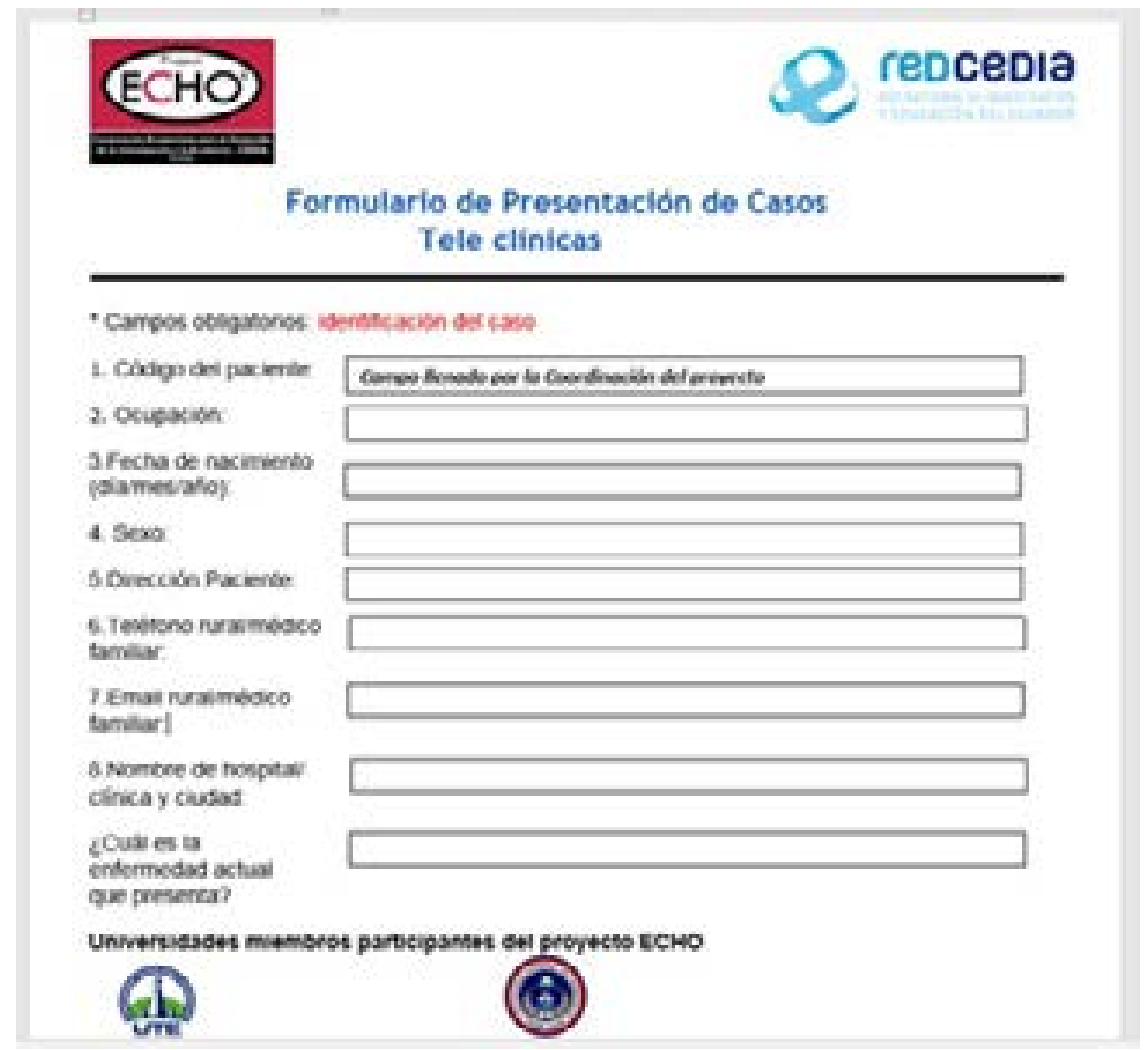

Figura 2. Página frontal del Formulario de presentación de casos de Teleclínicas. Ecuador, 2018.

Los participantes tienen acceso al link para la conexión a la reunión a través de la plataforma zoom, en el horario programado (Figura 3).

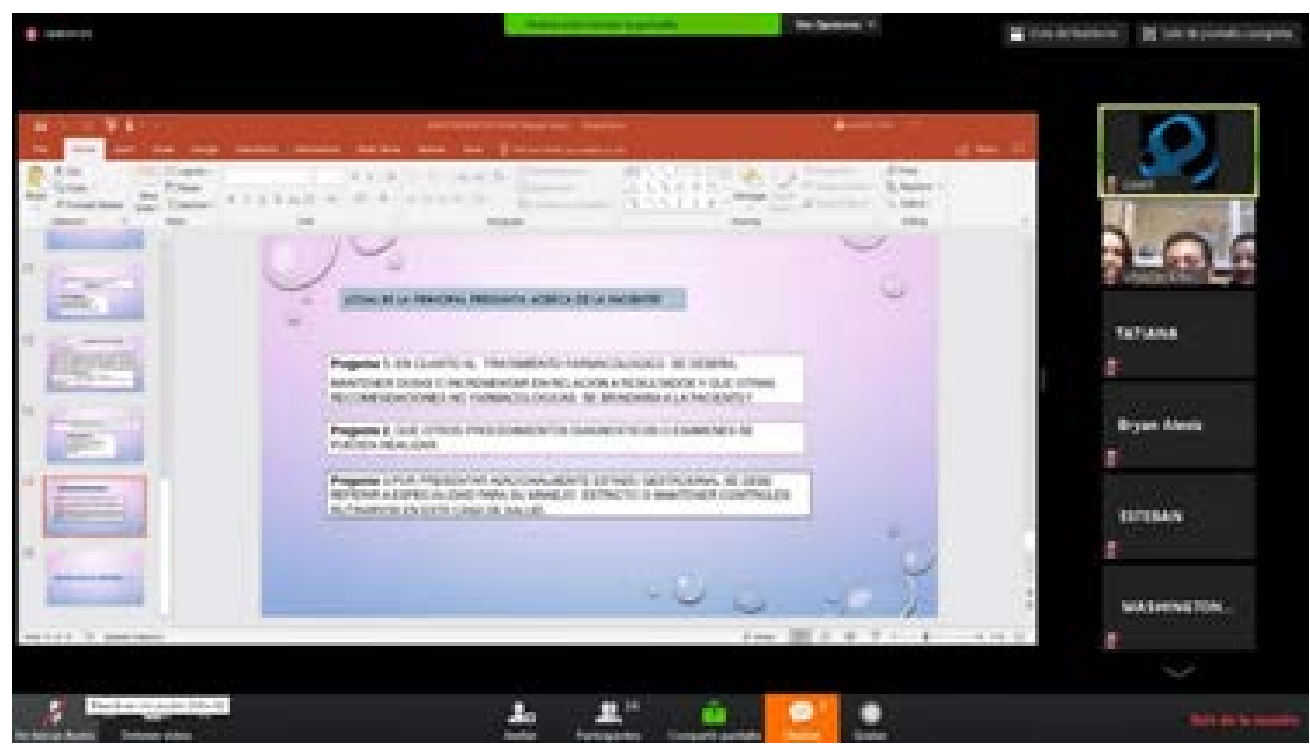

Figura 3. Sesión de teleclínica Proyecto ECHO a través de la plataforma ZOOM. Ecuador, 2018.

Fuente: Videoconferencia CEDIA -UNACH 
Para realizar la investigación, se definió un estudio cuantitativo, descriptivo, transversal, de campo y no experimental, con el fin de evaluar el grado de satisfacción de los participantes. Como se trata de una actividad de tipo virtual, la población de estudio consistió en 57 profesionales de diferentes unidades de salud, ubicadas en 15 provincias distribuidas en las cuatro regiones del país. La investigación se realizó durante el año 2018.

El cuestionario de encuesta se estructuró con 20 preguntas cerradas, así como datos generales. Se aplicó por medio de soporte digital de uso gratuito. 57 participantes, 139 médicos rurales, 8 especialistas en medicina familiar recién graduados, y 10 profesionales médicos varios), realizaron las encuestas de satisfacción.

Se agruparon las preguntas y respuestas, en las siguientes dimensiones: Accesibilidad, Metodología, Comunicación y desenvolvimiento durante las sesiones, Resolución de los temas tratados, Contribución al desarrollo profesional y aprendizaje a bajo costo, Contribución en la toma de decisiones y resolución de problemas.

Para le medición de las respuestas de la encuesta de satisfacción, se utilizó la Escala de Likert de satisfacción iniciando en 1 al máximo de 5 .

En tal virtud, a través de las encuestas, se ha determinado el nivel de satisfacción de los participantes con el sistema de teleclínicas del Proyecto ECHO, su aplicabilidad y contribución en la solución de problemas médicos.

\section{RESULTADOS}

Se presenta en primer lugar una visión global de los resultados de las respuestas proporcionadas por los profesionales participantes en la capacitación de teleclínicas, en los cinco niveles propuestos: "Muy de acuerdo", "De acuerdo", "Neutro", “En desacuerdo", “Muy en desacuerdo" (Figura 4)

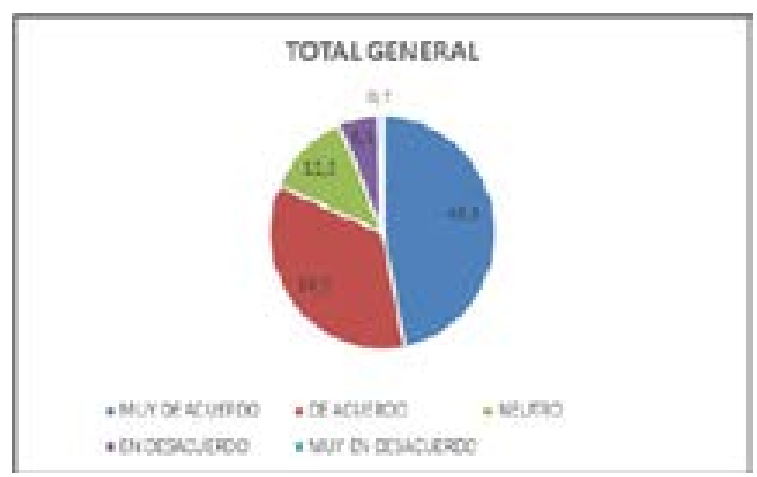

Figura 4. Resultados globales del estudio Fuente: Elaboración propia

Se puede observar que la mayoría de participantes $(81,5 \%)$ estuvieron satisfechos con 221 la experiencia.

En lo que respecta a las puntuaciones medias obtenidas en cada una de las dimensiones, en la tabla 1 se presentan dichos valores. Para una apropiada comprensión, debe considerarse que las alternativas de contestación fueron: "Muy de acuerdo" (5), “De acuerdo" (4), “Neutro" (3), “En desacuerdo" (2), "Muy en desacuerdo" (1). 


\begin{tabular}{|l|l|l|}
\hline DIMENSIÓN & MEDIA & DESV. EST. \\
\hline & & \\
\hline Accesibilidad & & \\
\hline Metodología & 4,05 & 0,84 \\
\hline Comunicación y desenvolvimiento durante las sesiones & 4,02 & 0,9 \\
\hline Resolución de los temas tratados & 4,11 & 0,83 \\
\hline Contribución al desarrollo profesional y aprendizaje a bajo costo & 4,38 & 1,00 \\
\hline Contribución en la toma de decisiones y resolución de problemas & 4,31 & 0,96 \\
\hline
\end{tabular}

Tabla 1. Valores medios obtenidos en las dimensiones evaluadas. Ecuador, 2018.

DESV. EST.: Desviación Estándar.

Fuente: Elaboración propia.

Se puede notar que todas las dimensiones tuvieron puntuaciones por encima de 4, reflejando un alto grado de satisfacción de los médicos participantes, en cuanto a los aportes y desarrollo de las actividades relacionadas con las teleclínicas.
En la tabla 2 se encuentran desglosados cada uno de los aspectos investigados en la encuesta, con sus respectivas puntuaciones alcanzadas. Los diferentes ítems han sido organizados conforme a la dimensión a la cual contribuyen.

\begin{tabular}{|c|c|c|}
\hline ESTRUCTURACIÓN POR DIMENSIONES & MEDIA & DESV. EST. \\
\hline DIMENSIÓN: Accesibilidad & & \\
\hline $\begin{array}{c}\text { ¿La calidad de la comunicación durante la sesión de teleclínica fue } \\
\text { adecuada? }\end{array}$ & 3,73 & 0,57 \\
\hline $\begin{array}{c}\text { ¿Prefiere tener una reunión de teleclínica desde su unidad que asistir a } \\
\text { una sesión de caso clínico en el hospital? }\end{array}$ & 3,98 & 0,75 \\
\hline ¿Está dispuesto (a) a tener presentaciones por teleclínica nuevamente? & 4,45 & 1,18 \\
\hline DIMENSIÓN: Metodología & & \\
\hline ¿El tiempo destinado para tratar el caso clínico fue adecuado para cubrir \\
todo lo requerido? & 4,19 & 0,89 \\
\hline ¿Está satisfecho (a) con el desarrollo de la sesión? & 4,29 & 0,96 \\
\hline ¿Fue capaz de hablar y dar toda la información que quería transmitir? & 3,92 & 0,70 \\
\hline
\end{tabular}




\begin{tabular}{|c|c|c|}
\hline $\begin{array}{l}\text { ¿La sesión de teleclínica observa los principios y criterios de ética } \\
\text { profesional? }\end{array}$ & 3,66 & 1,06 \\
\hline \multicolumn{3}{|l|}{ DIMENSIÓN: Comunicación y desenvolvimiento durante las sesiones } \\
\hline $\begin{array}{c}\text { ¿Considera que los médicos especialistas y demás participantes prestaron } \\
\text { suficiente atención en el caso clínico? }\end{array}$ & 4,19 & 0,88 \\
\hline ¿Se sintió apoyado(a) y alentado(a) en esta sesión? & 4,19 & 0,82 \\
\hline $\begin{array}{c}\text { ¿Se sintió cómodo(a) al comunicarse con el médico especialista por } \\
\text { teleconferencia en tiempo real? }\end{array}$ & 4,31 & 1,00 \\
\hline $\begin{array}{c}\text { ¿La sesión por teleclínica le permitió compartir con los colegas } \\
\text { especialistas en forma directa y amena? }\end{array}$ & 4,31 & 0,97 \\
\hline $\begin{array}{l}\text { ¿Durante la teleconferencia del caso clínico estuvo preocupado (a) por que } \\
\text { otros pudieran escucharle o verle? }\end{array}$ & 3,54 & 0,48 \\
\hline \multicolumn{3}{|l|}{ DIMENSIÓN: Resolución de los temas tratados } \\
\hline $\begin{array}{c}\text { ¿Las indicaciones y asesoría realizadas por los médicos especialistas } \\
\text { fueron claramente comprensibles? }\end{array}$ & 4,40 & 1,05 \\
\hline $\begin{array}{l}\text { En general, ¿está satisfecho (a) con la calidad de consultoría que se prestó } \\
\qquad \text { por teleclínica? }\end{array}$ & 4,28 & 0,94 \\
\hline \multicolumn{3}{|l|}{$\begin{array}{l}\text { DIMENSIÓN: Contribución al desarrollo profesional y aprendizaje a bajo } \\
\text { costo }\end{array}$} \\
\hline $\begin{array}{c}\text { ¿Considera que las sesiones de teleclínica contribuyen al desarrollo } \\
\text { profesional y la capacitación en el área médica? }\end{array}$ & 4,47 & 1,22 \\
\hline $\begin{array}{l}\text { ¿Encuentra la teleclínica una vía aceptable para compartir conocimientos } \\
\qquad \text { en la atención en salud? }\end{array}$ & 4,49 & 1,16 \\
\hline $\begin{array}{l}\text { ¿Con una sesión de teleclínica considera que existe un ahorro económico } \\
\text { en el sistema de salud comparada con una interconsulta convencional? }\end{array}$ & 4,17 & 0,91 \\
\hline \multicolumn{3}{|l|}{$\begin{array}{l}\text { DIMENSIÓN: Contribución en la toma de decisiones y resolución de } \\
\text { problemas }\end{array}$} \\
\hline $\begin{array}{l}\text { ¿La teleclínica extiende el acceso a los servicios de salud para los } \\
\text { pacientes en forma indirecta? }\end{array}$ & 4,28 & 0,92 \\
\hline $\begin{array}{l}\text { ¿Siente mayor seguridad y menos stress para tomar decisiones después } \\
\text { de la consulta del caso clínico del paciente con los médicos especialistas? }\end{array}$ & 4,35 & 0,99 \\
\hline $\begin{array}{c}\text { ¿En las sesiones de teleclínica obtuvo orientación que aporte en la } \\
\text { solución de problemas de la práctica médica? }\end{array}$ & 4,29 & 0,97 \\
\hline
\end{tabular}

Tabla 2. Valores medios obtenidos en cada uno de los ítems. Ecuador, 2018.

DESV. EST.: Desviación Estándar.

Fuente: Elaboración propia 
Lamayoríademédicosparticipantesconsideraron que las sesiones de teleclínica ofrecen un medio aceptable para compartir conocimientos de la atención de salud, evidenciándose un puntaje de 4,49; en la pregunta que evalúa la contribución al desarrollo profesional y capacitación médica que ofrece este sistema de teleclínicas, los resultados fueron elevados $(4,47)$; los asistentes a estas sesiones virtuales percibieron que la comprensibilidad de las indicaciones y asesoría de los especialistas es alta, con una media de 4,40. Estos valores alcanzados corresponden a ítems de las categorías "Resolución de los temas tratados" y "Contribución al desarrollo profesional y aprendizaje a bajo costo", lo que se relaciona con la percepción positiva de los profesionales, en cuanto al ahorro económico que representa en el sistema de salud, la realización de una sesión de teleclínica frente a una interconsulta convencional.

En las dimensiones de "Accesibilidad" y "Metodología" se obtuvieron respuestas de valoración intermedia. Como ejemplo, en la pregunta “¿La calidad de la comunicación durante la sesión de teleclínica fue adecuada?" y ¿Prefiere tener una reunión de teleclínica desde su unidad que asistir a una sesión de caso clínico en el hospital?, se alcanzó puntuaciones que se ubicaron ligeramente por debajo de la media. De igual forma, en los siguientes ítems: “¿Fue capaz de hablar y dar toda la información que quería transmitir? “ ¿La sesión de teleclínica observa los principios y criterios de ética profesional?". Esto demuestra, que los asistentes estuvieron satisfechos con la metodología utilizada.

La dimensión "Comunicación y desenvolvimiento durante las sesiones" estuvo enfocada en la percepción del participante sobre la facilidad de interacción con los médicos especialistas y el resto de asistentes a las sesiones virtuales. El valor más bajo se encontró en el ítem “¿Durante la teleconferencia del caso clínico estuvo preocupado (a) por que otros pudieran escucharle o verle?" $(3,54)$. Al mismo tiempo, este valor contrasta con puntajes alcanzados en la dimensión "Accesibilidad", en la cual se evidencia valores superiores a la media de la categoría, en la pregunta “ ¿Está dispuesto (a) a tener presentaciones por teleclínica nuevamente?", con un puntaje medio de 4.45 , es decir entre los más altos del estudio.

Uno de los fines globales del proyecto es contribuir en la solución de problemas en el manejo de los pacientes, mediante la capacitación y tutoría impartida a médicos de áreas rurales. Por tal motivo, la evaluación de los aspectos incluidos en la última dimensión de la encuesta, es decir, "Contribución en la toma de decisiones y resolución de problemas", es de gran relevancia, ya que los valores se acercaron al puntaje máximo en todos los aspectos.

\section{DISCUSIÓN}

Los resultados reflejan que el $81,5 \%$ de los profesionales de salud estuvieron de acuerdo con la aplicación de esta metodología de tipo colaborativa, a través de medios virtuales, como una herramienta útil en su desarrollo y crecimiento profesional, que se extiende a la posibilidad de alcanzar una mejor condición de salud y calidad de vida de la población; situación que fue evaluada en el año 2018, durante el cual se realizaron cuarenta sesiones de teleclínica.

Es pertinente señalar como referente que, desde fines de 2015, Uruguay constituye el primer superhub o centro de referencia del Proyecto ECHO, en América Latina. En este país se desarrollan numerosas teleclínicas en diferentes áreas y enfocadas en diversas patologías. En este mismo año, ECHO Uruguay fue señalado de interés ministerial y en 2016, de interés nacional (111. En relación a este trabajo, existe un estudio efectuado en la Clínica Ginecotocológica A de la Facultad de Medicina, en donde se realizaron las teleclínicas ECHO para el manejo de la prevención y el tratamiento 
del cáncer de cuello uterino (CCU) en el período junio de 2015 a julio de 2016 con un total de 13 sesiones; los resultados indicaron que el proyecto ha coadyuvado en la homogenización de criterios diagnósticos, además de mantener actualizados todos los aspectos de la patología (Nozar, y otros, 2017).

En el mismo sentido, a través de la presente investigación, se encontró un alto nivel de satisfacción de los participantes en las teleclínicas de endocrinología e hipertensión, en lo concerniente al desarrollo, metodología, contribución para la capacitación médica a bajo costo y en la seguridad en la toma de decisiones, en especial a aquellos profesionales de atención primaria y que se encuentran alejados de los centros médicos más desarrollados.

Como limitación del estudio, el $43.8 \%$ de los participantes revelaron haber tenido inconvenientes para la conexión, lo que se atribuye a una desigualdad en el acceso a internet, especialmente por tratarse de zonas rurales.

De igual forma, los resultados más bajos obtenidos en los ítems de las categorías de “Accesibilidad” y “Metodología”, servirán de guía a los organizadores, en la adecuación de mejoras para la accesibilidad y desarrollo de la reunión.

En conclusión, desde la percepción de los profesionales, la aplicación del proceso de enseñanza a distancia y teletutoría del Proyecto ECHO (teleclínicas), les brindó un soporte de aprendizaje permanente, que contribuyó a la solución de problemas de la práctica médica de atención primaria y general, así como el manejo de los pacientes, evidenciando un alto grado de satisfacción profesional.

La capacitación a través de las asesorías virtuales cooperativas y la comunicación en tiempo real, disminuyó la sensación de aislamiento, en tal virtud, los profesionales de salud se mostraron predispuestos a continuar participando en las reuniones. Los médicos rurales se sintieron más cómodos y seguros en sus actividades y en la toma de decisiones, respecto al tratamiento y seguimiento de sus pacientes.

Mediante esta investigación, se demostró que las sesiones de teleclínica, lograron satisfacer los requerimientos de aprendizaje y asesoría para médicos rurales a poco costo, lo que repercutirá en la calidad de atención oportuna a las poblaciones de áreas remotas y desatendidas, cumpliendo así con los principios del modelo ECHO.

Asimismo, estos resultados constituyen una evidencia para sustentar la implementación de los medios virtuales y telemedicina en las políticas públicas de atención y capacitación en salud en Ecuador y Latinoamérica.

Agradecimientos: Los autores expresan el reconocimiento a la Corporación Ecuatoriana para el Desarrollo de la Investigación y la Academia CEDIA y a la Universidad Nacional de Chimborazo, instituciones partícipes del Convenio respectivo.

Conflicto de intereses: El Proyecto ECHO es un programa de aprendizaje profesional (ECHO Model TM) para proveedores de salud en áreas rurales, creado por el Instituto ECHO (Universidad de New México, Facultad de Medicinal, en Albuquerque, USA. Este artículo se basa en un trabajo de investigación relacionado con un Convenio para el desarrollo del Proyecto ECHO, entre la Corporación Ecuatoriana para el Desarrollo de la Investigación y la academia CEDIA y la Universidad Nacional de Chimborazo 2018. Mediante un acuerdo, CEDIA también obtuvo los derechos y la licencia de la Universidad de Nuevo México, para reproducir el Proyecto ECHO en Ecuador. 


\section{REFERENCIAS BIBLIOGRÁFICAS}

Arora, S., Thornton, K., Murata, G., Deming, P., Kalishman, S., Dion, D., . . Qualls, C. (1 de junio de 2011). Outcomes of Treatment for Hepatitis C. The New England Journal of Medicine, 364(23), 2199-2207. Recuperado el 10 de septiembre de 2019, de https://www. nejm.org/doi/pdf/10.1056/NEJMoa1009 370 ?articleTools=true $\&$ downloadfile $=$ sh owPdf\&articleTools=true \&doi=10.1056/ NEJMoa1009370

Asamblea Consitituyente. (2008). Consititución del Ecuador. Registro Oficial 449. Recuperado el 17 de septiembre de 2019, de http://www.oas.org/juridico/ pdfs/mesicic4_ecu_const.PDF

Cabero-Almenara, J., Llorente-Cejudo, M., \& Puentes-Puente, Á. (2010). Online students' satisfaction with blended tearning La satisfacción de los estudiantes en red en la formación semipresencial). Comunicar, 35, 149157. doi:https://doi.org/10.3916/C352010-03-08

Cohen, H. (18 de marzo de 2019). proyecto echo: ies posible democratizar la atención médica en américa latina? Acta Gastroenterol Latinoam, 49(1), 6-10. Recuperado el 10 de septiembre de 2019 , de https://actagastro.org/proyectoecho-es-posible-democratizar-laatencion-medica-en-america-latina/

Comisión Económica para América Latina y el Caribe CEPAL. (2018). Anuario Estadístico de América Latina y el Caribe. Recuperado el 17 de septiembre de 2019, de https://www.cepal.org/es/ publicaciones/ae

Cordero Torres, J. A., \& Caballero Oliver, A. (2015). La plataforma Moodle: Una herramienta útil para la formación en soporte vital. Análisis de las encuestas de satisfacción a los alumnos e instructores de los cursos de soporte vital avanzado del programa ESVAP de la semFYC. Atención Primaria, 47(6), 376-384. doi:http://dx.doi.org/10.1016/j. aprim.2015.02.006

Corporación Ecuatoriana para el Desarrollo de la Investigación y la Academica CEDIA. Universidad Nacional de Chimborazo UNACH. (2018). Convenio Específico para el Desarrollo del Proyecto ECHO entre la Fundación Consorcio para el Desarrollo de internet Avanzado CEDIA y la Universidad Nacional de Chimborazo. Recuperado el 23 de septiembre de 2019, de Uiversidad Nacional de Chimborazo Report No.: COC-AL-08-18: http://www.unach.edu. ec/images/galeriajulio/convenioslibros/ nacionales/2018/Enero/2018 - ENE - 18 CONVENIO COC-AL-08-18 ECHO.pdf

Díaz, K., Miguel, V., Landaeta, I., Ortiz, M., \& Fernández, M. (2014). atisfacción Estudiantil con el uso de Estrategias Instruccionales Apoyadas en Tic para el Aprendizaje de Bioquímica. Docencia Univ, 15(2), 2-16. Recuperado el 6 de mayo de 2019, de http://saber.ucv. ve/ojs/index.php/rev_docu/article/ view/8467/8359

Herrero Martínez, R. (2014). El papel de las TIC en el aula universitaria para la formación en competencias del alumnado. Revista de Medios y Educación, 45(45), 173-188. doi:10.12795/pixelbit.2014.i45.12

Instituto Nacional de Estadísticas y Censos INEC. (2016). Registro Estadístico de Recursos y Actividades de Salud - RAS 2016. Recuperado el 17 de septiembre de 2019, de https://www.ecuadorencifras. 
gob.ec/documentos/web-

Lascano Soriano, L. D., \& Sánchez Castro, X. (Agosto de 2018). Investigación acerca de la factibilidad de la Telemedicina en el área de Psiquiatría en la Clínica Santa Isabel. (U. d. Guayaquil, Ed.) Guayaquil, Guayas, Ecuador. Recuperado el 17 de septiembre de 2019, de http:// repositorio.ug.edu.ec/bitstream/ redug/37003/1/TESIS LASCANO LUIS \%28FINAL\%29.pdf

Marín Díaz , V., Reche Urbano, E., \& Maldonado Berea, G. A. (Diciembre de 2013). Advantages and disadvantages of online training. Rev Digit Investig en Docencia Univ RIDU, 7(1), 33-43. Recuperado el 7 de mayo de 2019, de https://dialnet.unirioja.es/servlet/ articulo?codigo $=4775417$

Maruri Sigüenza, J. C., \& Castro Rugel , L. (Agosto de 2016). PLAN DE TELEMEDICINA PARA MEJORAR LA ATENCIÓN. IU. d. Guayaquil, Ed.) Guayaquil, Guayas, Ecuador. Recuperado el 17 de septiembre de 2019, de http://repositorio.ug.edu.ec/ bitstream/redug/15773/1/TESIS_Juan Carlos Maruri Siguenza.pdf

Naranjo Ferregut, J. A., Delgado Cruz, A., Rodríguez Cruz, R., \& Sánchez Pérez, Y. (2014). Consideraciones sobre el Modelo de Atención Integral. Revista Cubana de Medicina General Integral, 30(3), 375381. Recuperado el 17 de septiembre de 2019, de http://scielo.sld.cu/pdf/mgi/ v30n3/mgi11314.pdf

Nozar, M., Fiol, V., Greif , D., Ben , S., Briozzo, L., \& Cohen, H. (2017). áncer de cuello uterino, estrategias innovadoras en la descentralización del conocimiento y su manejo. Proyecto ECHO Uruguay. Revista Médica del Uruguay, 33(1), 128-141.
Recuperado el 12 de septiembre de 2019, de http://www.scielo.edu.uy/scielo. php?script=sci_arttext $\&$ pid $=$ S1688$03902017000100128 \& \operatorname{lng}=e s \&$ thng=es

Organización Mundial de la Salud. (2006). Trabajar en pro de la salud: presentación de la Organización Munidal de la Salud. Recuperado el 23 de septiembre de 2019, de https://www.who.int/about/ brochure_es.pdf?ua=1

Ramón, H., Russo, C., Sarobe, M., Alonso, N., Esnaola, L., Ahmad, T., \& Padovani, F. (Abril de 2014). El uso de los Entornos Virtuales 3D como. (R. d.-U. UNLP), Ed.) Revista Iberoamericana de Educación en Tecnología y Tecnología en Educación Especial(12), 72-80. Recuperado el 6 de noviembre de 2018, de http://sedici.unlp.edu.ar/bitstream/ handle/10915/36009/Documento_ completo. pdf?sequence $=1$ \&isAllowed $=y$

University of New Mexico. (2019). ECHO's impact. Recuperado el 23 de septiembre de 2019, de https://echo.unm.edu/echosimpact

University of New Mexico. School of Medicine. (2018). Project ECHO. Obtenido de https://echo.unm.edu/old

Zoom Video Commuications, Inc. (2019). Zoom Video Commuications. Recuperado el 24 de septiembre de 2019, de https://zoom. us/es-es/meetings.html 